\title{
Structure and kinematics of the Louth-Eumarra Shear Zone (north- central New South Wales, Australia) and implications for the Paleozoic plate tectonic evolution of eastern Australia
}

\author{
S. DUNSTAN, G. ROSENBAUM AND A. BABAAHMADI
}

School of Earth Sciences, The University of Queensland, Brisbane 4072, Australia

*Corresponding author (Ph: 073346 9798, Email: g.rosenbaum@uq.edu.au)

\section{SUPPLEMENTARY PAPERS}

Australian Journal of Earth Sciences (2016) 63 (1)

http://dx.doi.org/10.1080/08120099.2016.1131195

Copies of Supplementary Papers may be obtained from the Geological Society of Australia's website (www.gsa.org.au), the Australian Journal of Earth Sciences website (www.ajes.com.au) or from the National Library of Australia's Pandora archive (http://nla.gov.au/nla.arc-25194).

\section{SUPPLEMENTARY PAPERS}

A1. Field and drill core sample information table.

A2. SEM-BSE images and SEM-EDS spectra from sample 14-SD-03 collected from drill core DDHTE Templestone Anomaly $\left(-30.215023^{\circ} \mathrm{S}, 146.608217^{\circ} \mathrm{E}\right), 115.9$ $m$ depth. 
Dunstan et al. 2016. Australin Journal of Earth Sciences 63 http://dx.doi.org/10.1080/08120099.2016.1131195

A1. Field and drill core sample information table.

\begin{tabular}{|c|c|c|c|c|c|c|}
\hline Sample & Latitude & Longitude & Sample type & Drill hole/Depth & Lithology & Comments \\
\hline 14-SD-25 & $30.46645^{\circ} \mathrm{S}$ & $146.68734^{\circ} \mathrm{E}$ & Field sample & - & Quartzite & Weak foliation developed (S2). \\
\hline 14-SD-03 & $30.21502^{\circ} \mathrm{S}$ & $146.608217^{\circ} \mathrm{E}$ & Drill core & $\begin{array}{l}\text { DDHTE } \\
\text { Templestone } \\
\text { Anomaly/ } 115.9 \mathrm{~m}\end{array}$ & $\begin{array}{l}\text { Biotite- } \\
\text { muscovite schist }\end{array}$ & $\begin{array}{l}\text { Two foliations. Strongly developed S2 crenulation cleavage } \\
\text { with remnant S1 within microlithons of S2. }\end{array}$ \\
\hline 14-SD-06 & $30.33722^{\circ} \mathrm{S}$ & $146.139375^{\circ} \mathrm{E}$ & Drill core & $\begin{array}{l}\text { DDH-01 } \\
\text { Woodstock/ } 151 \mathrm{~m}\end{array}$ & Mica schist & $\begin{array}{l}\text { Two foliations. S2 foliation within sample occurring axial } \\
\text { planar to F2 folds, remnant S1 slaty cleavage in microlithons. }\end{array}$ \\
\hline 14-SD-07 & $30.35503^{\circ} \mathrm{S}$ & $146.314388^{\circ} \mathrm{E}$ & Drill core & $\begin{array}{l}\text { DR20 Doradilla/ } \\
159.1 \mathrm{~m}\end{array}$ & Muscovite schist & $\begin{array}{l}\text { Two foliations. Strongly developed S2 crenulation cleavage } \\
\text { with development of quartz-mica domains. Remnant S1 } \\
\text { found within microlithons of S2. }\end{array}$ \\
\hline 14-SD-08 & $30.174047^{\circ} \mathrm{S}$ & $146.671944^{\circ} \mathrm{E}$ & Drill core & $\begin{array}{l}\text { BO-1 Boomi/ } \\
133.4-133.5 \mathrm{~m}\end{array}$ & $\begin{array}{l}\text { Biotite- } \\
\text { muscovite schist }\end{array}$ & $\begin{array}{l}\text { Only one foliation weakly developed in sample. Growth of } \\
\text { chlorite and biotite along foliation planes. }\end{array}$ \\
\hline 14-SD-01 & $30.322747^{\circ} \mathrm{S}$ & $146.405670^{\circ} \mathrm{E}$ & Drill core & $\begin{array}{l}\text { DDHTE-1 } \\
\text { Templestone } \\
\text { anomaly/ 142.9- } \\
143 \mathrm{~m}\end{array}$ & $\begin{array}{l}\text { Biotite- } \\
\text { muscovite schist }\end{array}$ & $\begin{array}{l}\text { Three foliations. S1 found within microlithons of strongly } \\
\text { developed S2 crenulation cleavage. S3 is a weakly } \\
\text { developed (incipient) crenulation cleavage. }\end{array}$ \\
\hline 14-SD-11 & $30.322747^{\circ} \mathrm{S}$ & $146.405670^{\circ} \mathrm{E}$ & Drill core & $\begin{array}{l}\text { DBT-1 Bobs Tank/ } \\
121.6-121.7 \mathrm{~m}\end{array}$ & $\begin{array}{l}\text { Biotite- } \\
\text { muscovite schist }\end{array}$ & $\begin{array}{l}\text { Foliation within this rock is defined by prominent quartz-mica } \\
\text { domains presumably following a strong crenulation cleavage. }\end{array}$ \\
\hline 14-SD-12 & $30.315920^{\circ} \mathrm{S}$ & $146.369827^{\circ} \mathrm{E}$ & Drill core & $\begin{array}{l}\text { MW2 Midway } \\
\text { Doradilla line/ } \\
98.9-99 \mathrm{~m}\end{array}$ & $\begin{array}{l}\text { Chlorite-biotite- } \\
\text { muscovite schist }\end{array}$ & $\begin{array}{l}\text { Two foliations. Dominant foliation within sample (S2?) } \\
\text { defined by quartz-mica domains overprinted by incipient } \\
\text { crenulation cleavage (S3?). }\end{array}$ \\
\hline 14-SD-14 & $30.286101^{\circ} \mathrm{S}$ & $146.423994^{\circ} \mathrm{E}$ & Drill core & $\begin{array}{l}\text { 3Kel-18 Doradilla } \\
\text { line }\end{array}$ & $\begin{array}{l}\text { Muscovite } \\
\text { phyllite }\end{array}$ & $\begin{array}{l}\text { Two foliations. Dominant foliation (S2?) defined tightly } \\
\text { spaced }(<1 \mathrm{~mm}) \text { quartz-mica domains. Overprinted by a } \\
\text { kinked crenulation cleavage (S3?). }\end{array}$ \\
\hline 14-SD-17 & $30.273041^{\circ} \mathrm{S}$ & $146.355557^{\circ} \mathrm{E}$ & Drill core & $\begin{array}{l}\text { Moocha MO-1/ } \\
229.5 \mathrm{~m}\end{array}$ & $\begin{array}{l}\text { Biotite- } \\
\text { muscovite schist }\end{array}$ & $\begin{array}{l}\text { One strong slaty cleavage in the sample. Quartz veins folded } \\
\text { around foliation. }\end{array}$ \\
\hline 14-SD-18 & $30.273041^{\circ} \mathrm{S}$ & $146.355557^{\circ} \mathrm{E}$ & Drill core & $\begin{array}{l}\text { Moocha MO-1/ } \\
170.8 \mathrm{~m}\end{array}$ & $\begin{array}{l}\text { Biotite- } \\
\text { muscovite schist }\end{array}$ & $\begin{array}{l}\text { One strong foliation defined by quartz-mica domains. No } \\
\text { evidence for secondary foliation found. }\end{array}$ \\
\hline 14-SD-20 & $30.180218^{\circ} \mathrm{S}$ & $146.617911^{\circ} \mathrm{E}$ & Drill core & $\begin{array}{l}\text { Frontage Tank/ } \\
166.6 \mathrm{~m}\end{array}$ & $\begin{array}{l}\text { Bioitite- } \\
\text { muscovite schist }\end{array}$ & $\begin{array}{l}\text { Two foliations. Strong foliation (S2?) defined by strongly } \\
\text { developed quartz-mica domains, which are overprinted by a } \\
\text { strong crenulation cleavage (S3?). }\end{array}$ \\
\hline T79665 & $30.347791^{\circ} \mathrm{S}$ & $146.295167^{\circ} \mathrm{E}$ & $\begin{array}{l}\text { GSNSW field } \\
\text { sample }\end{array}$ & - & $\begin{array}{l}\text { Biotite- } \\
\text { muscovite schist }\end{array}$ & $\begin{array}{l}\text { Dominant foliations (S2?) defined by quartz-mica domains, } \\
\text { which is overprint by an incipient crenulation cleavage (S3?). } \\
\text { A remnant S1 foliation is found in lower strain areas }\end{array}$ \\
\hline T79644 & $30.356676^{\circ} \mathrm{S}$ & $146.313178^{\circ} \mathrm{E}$ & $\begin{array}{l}\text { GSNSW drill } \\
\text { core sample }\end{array}$ & $\begin{array}{l}\text { DR20 Doradilla/ } \\
266.5 \mathrm{~m}\end{array}$ & $\begin{array}{l}\text { Biotite- } \\
\text { muscovite schist }\end{array}$ & $\begin{array}{l}\text { One major foliation in the rock defined by quartz-mica } \\
\text { domains. }\end{array}$ \\
\hline T79657 & $30.345777^{\circ} \mathrm{S}$ & $146.316926^{\circ} \mathrm{E}$ & $\begin{array}{l}\text { GSNSW drill } \\
\text { core sample }\end{array}$ & $\begin{array}{l}\text { YTC drillhole } \\
\text { DCMD001/ } 79 \mathrm{~m}\end{array}$ & Muscovite schist & $\begin{array}{l}\text { One major foliation defined by strongly developed quartz- } \\
\text { mica domains. }\end{array}$ \\
\hline
\end{tabular}




\begin{tabular}{|l|c|c|c|c|c|l|}
\hline Sample & Latitude & Longitude & Sample type & Drill hole/Depth & Lithology & \multicolumn{1}{c|}{ Comments } \\
\hline T79647 & $30.356676^{\circ} \mathrm{S}$ & $146.313178^{\circ} \mathrm{E}$ & $\begin{array}{l}\text { GSNSW drill } \\
\text { core sample }\end{array}$ & $\begin{array}{l}\text { DR20 Doradilla/ } \\
123 \mathrm{~m}\end{array}$ & Muscovite schist & $\begin{array}{l}\text { One major foliation (S2) within the rock defined by aligned } \\
\text { muscovite domains. Very rare occurrences of S1 in } \\
\text { microlithons of S2. }\end{array}$ \\
\hline 14-SD-23B & $31.177997^{\circ} \mathrm{S}$ & $146.843262^{\circ} \mathrm{E}$ & Field sample & - & Muscovite schist & $\begin{array}{l}\text { Up to 3 foliations. S2 foliations defined by quartz-mica } \\
\text { domains overprinted by a strong crenulation cleavage (S3). } \\
\text { S1 rarely found in microlithons of S2. }\end{array}$ \\
\hline $14-S D-29 \mathrm{~A}$ & $31.287321^{\circ} \mathrm{S}$ & $146.435049^{\circ} \mathrm{E}$ & Field sample & - & $\begin{array}{l}\text { Biotite- } \\
\text { muscovite schist }\end{array}$ & $\begin{array}{l}\text { Three foliations developed. Major foliation is S2, which is a } \\
\text { strongly developed crenulation cleavage. S1 found in } \\
\text { microlithons of S2. Very weak S3 foliation found overprinting } \\
\text { entire sample. }\end{array}$ \\
\hline $14-S D-30$ & $31.291474^{\circ} \mathrm{S}$ & $146.398281^{\circ} \mathrm{E}$ & Field sample & - & Muscovite schist & $\begin{array}{l}\text { Main foliation (S2?) defined by strong crenulation cleavage } \\
\text { forming quartz-mica domains. S1 foliation found in } \\
\text { microlithons of S2. }\end{array}$ \\
\hline $14-S D-31$ & $31.291919^{\circ} \mathrm{S}$ & $146.399699^{\circ} \mathrm{E}$ & Field sample & - & Muscovite schist & $\begin{array}{l}\text { Very close to sample 14-SD-30. Main foliation (S2?) defined } \\
\text { by strongly developed crenulation cleavage, which has } \\
\text { developed quartz-mica domains. Very incipient S3 foliation } \\
\text { throughout sample. }\end{array}$ \\
\hline
\end{tabular}




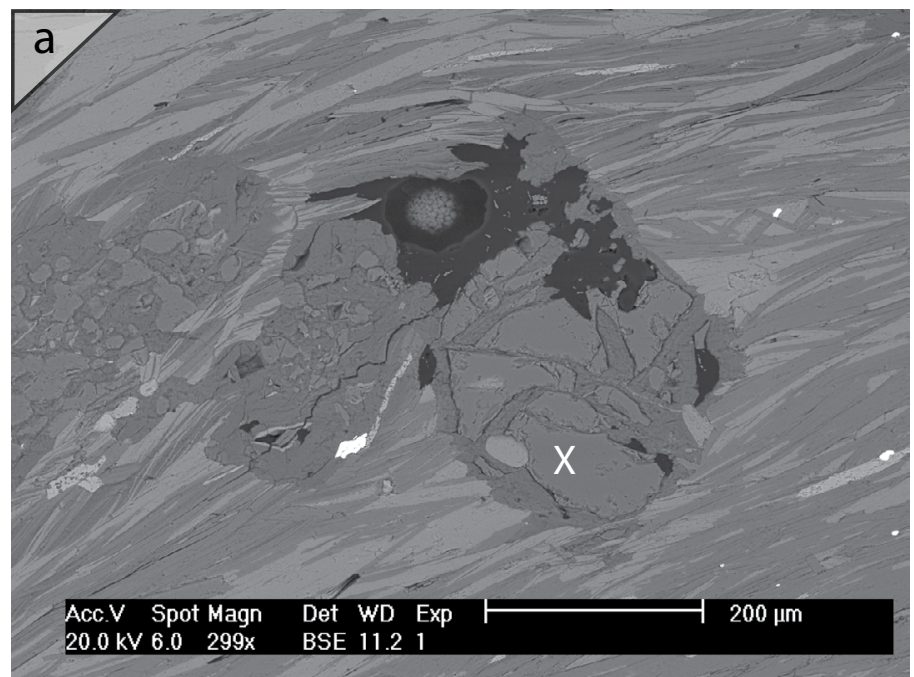

A2. a) SEM-BSE image of kyanite (as shown in Figure 7e)

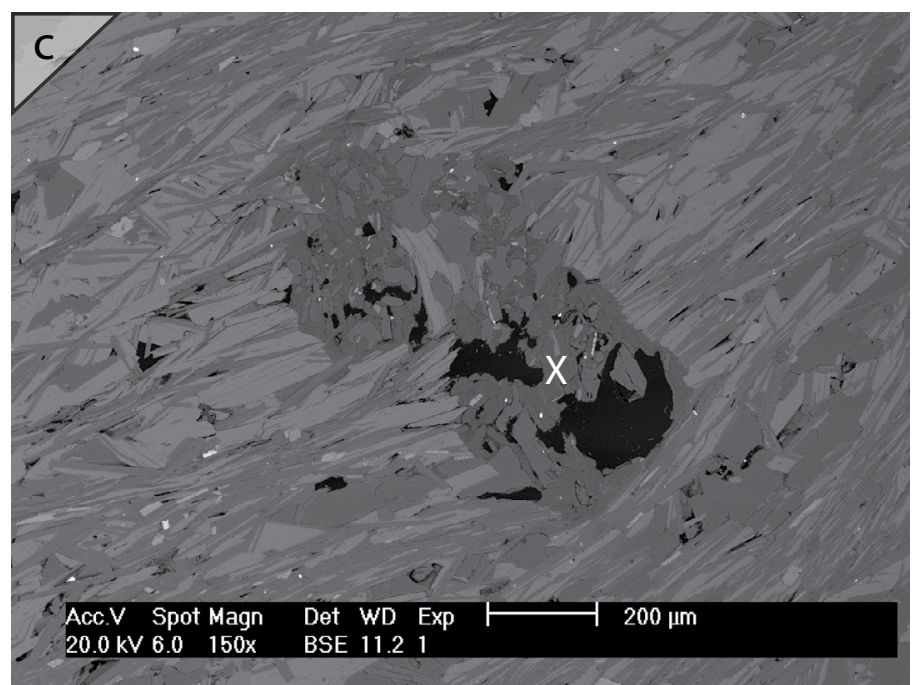

A2. c) SEM-BSE image of late stage mineral forming after the major deformation. Potentially cordierite.

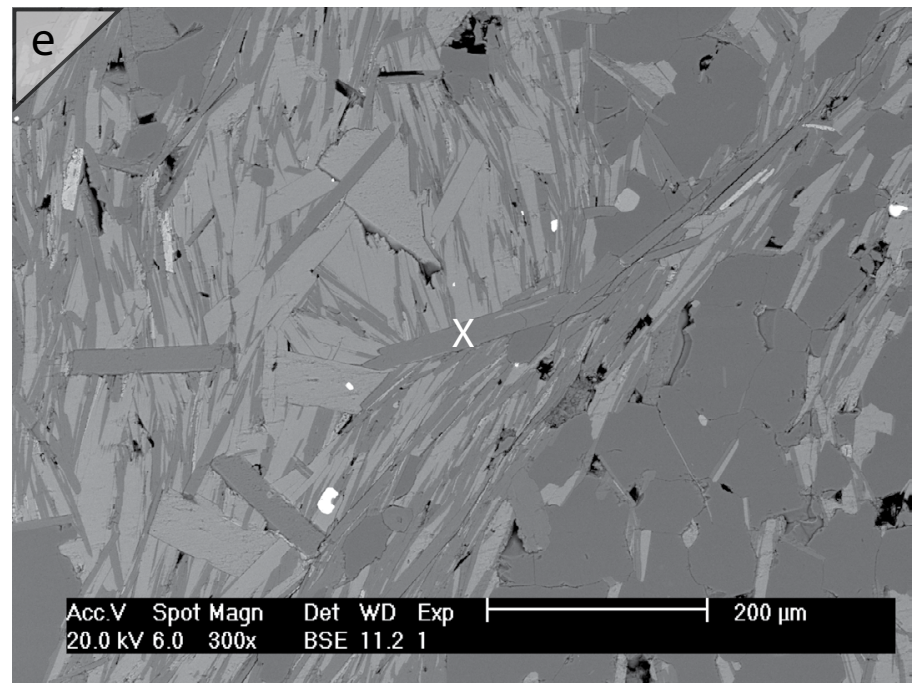

A2. e) SEM-BSE image metamorphic muscovite.

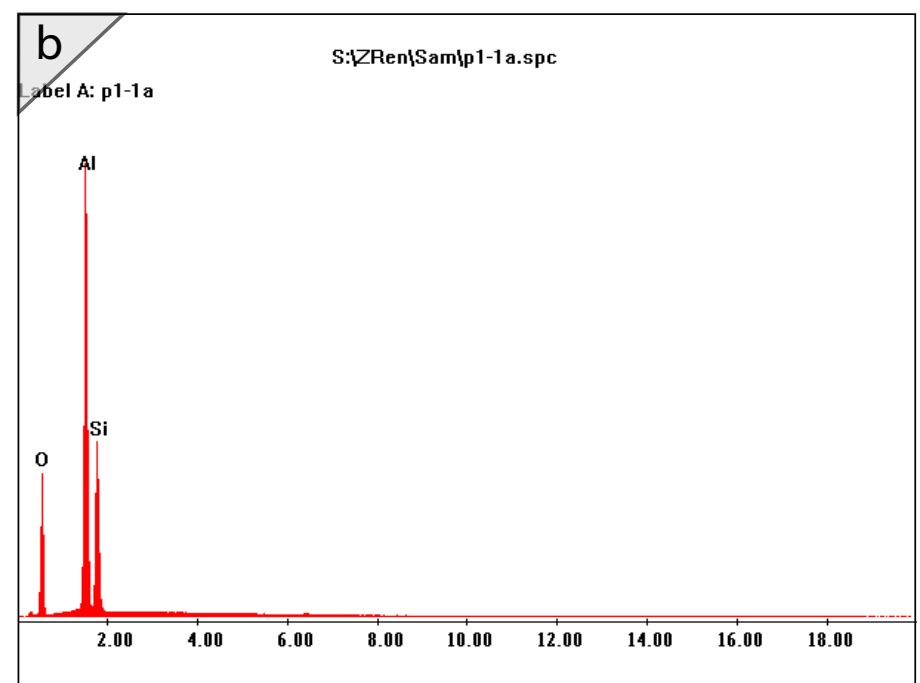

A2. b) SEM-EDS spectra of kyanite shown in A2. a).

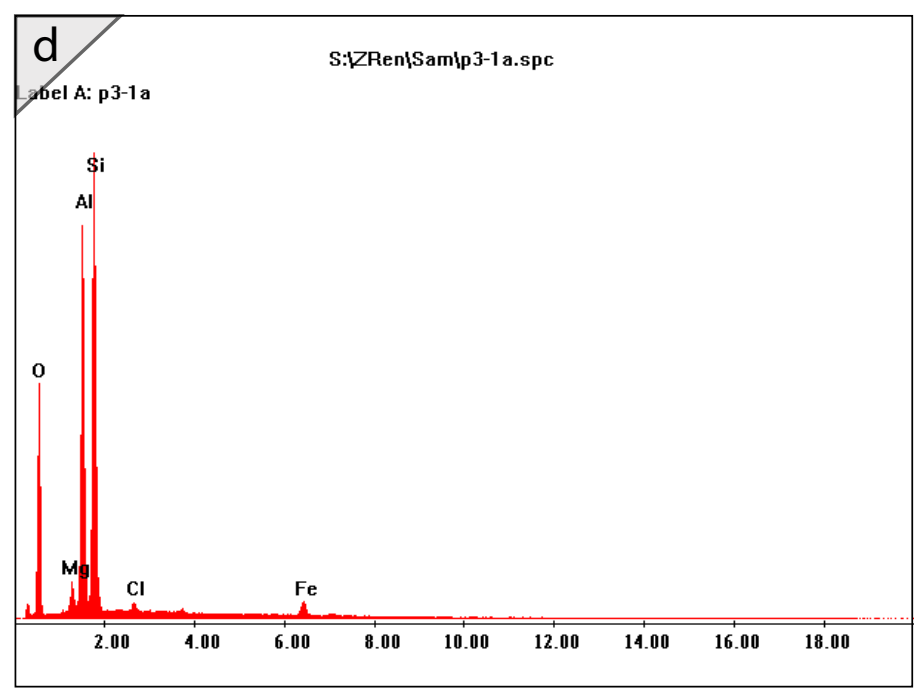

A2. d) SEM-EDS spectra the mineral in A2. c). The high aluminium peak and the minor magnesium peak indicate this mineral is potentially cordierite.

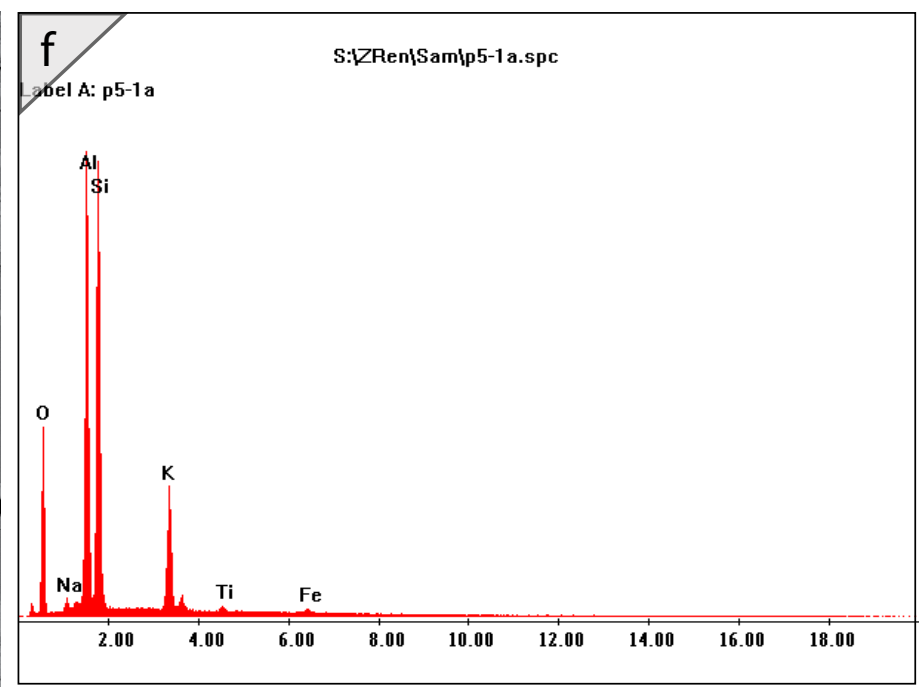

A2. f) SEM-EDS spectra of the muscovite in A2.e) 
Dunstan et al. 2016. Australin Journal of Earth Sciences 63

http://dx.doi.org/10.1080/08120099.2016.1131195
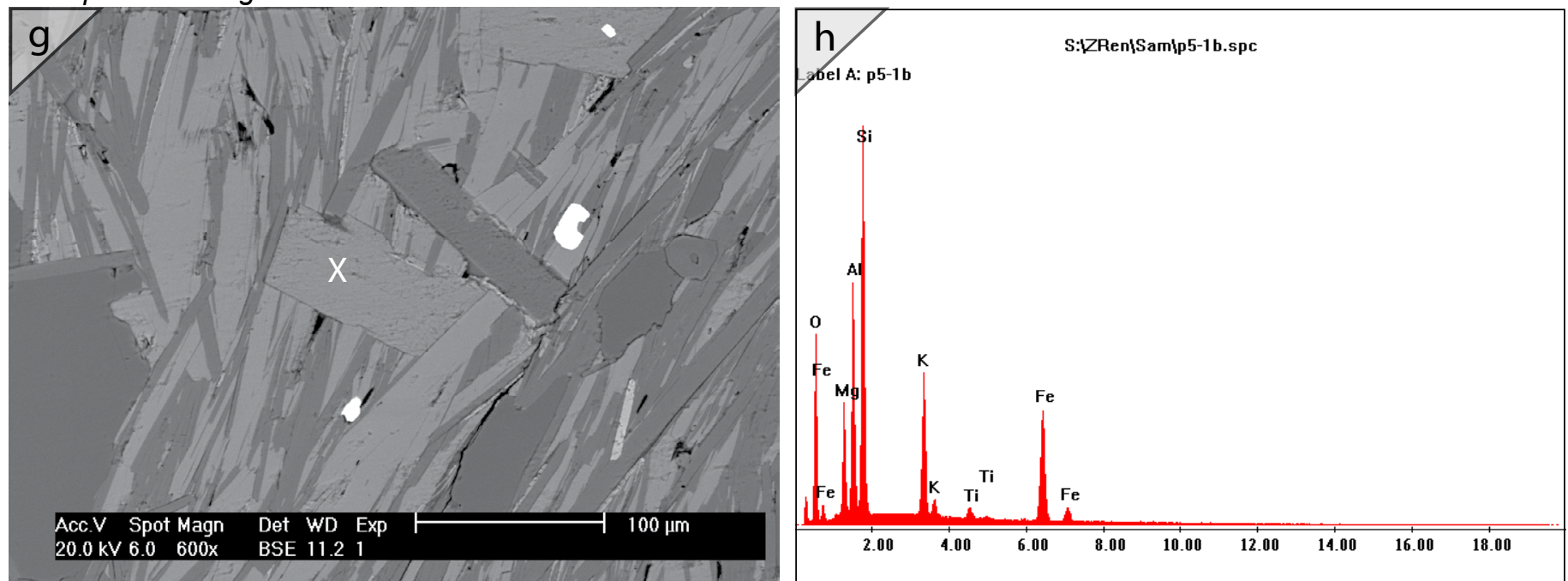

A2. g) SEM-BSE image of metamorphic biotite.

A2. h) SEM-EDS spectra of the biotite in A2.g)
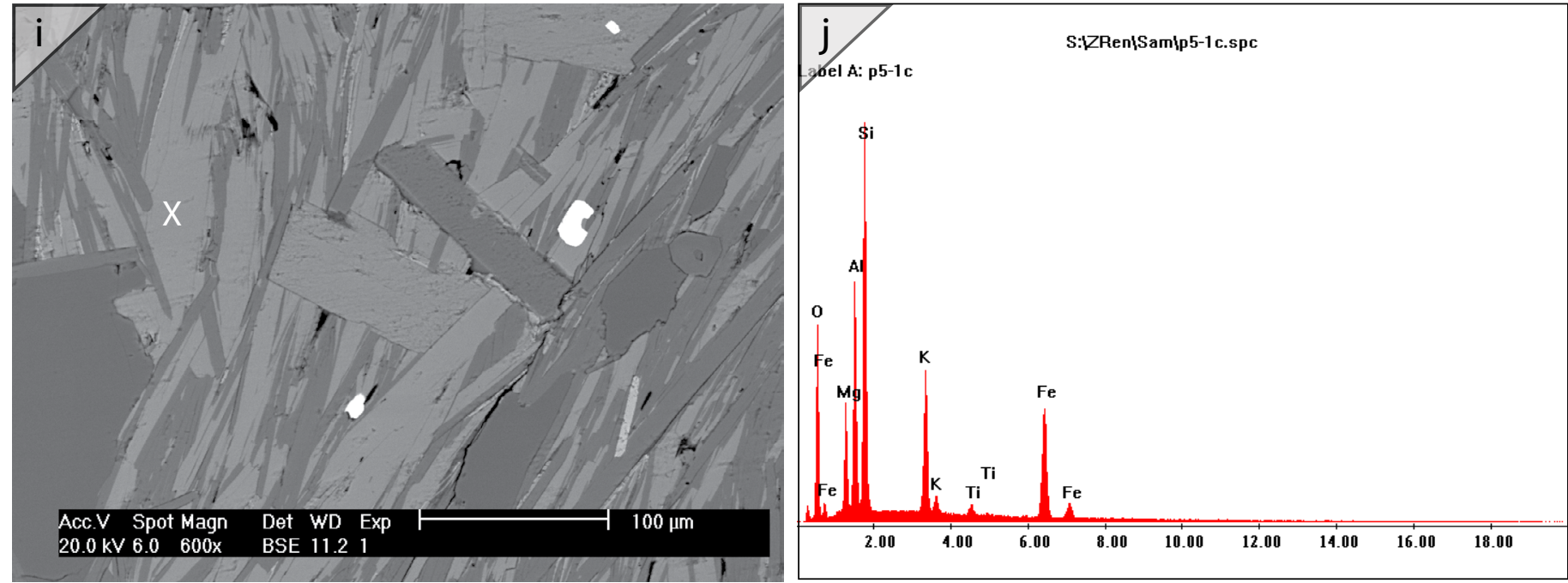

A2. g) SEM-BSE image of metamorphic biotite.

A2. h) SEM-EDS spectra of the biotite in A2.i)
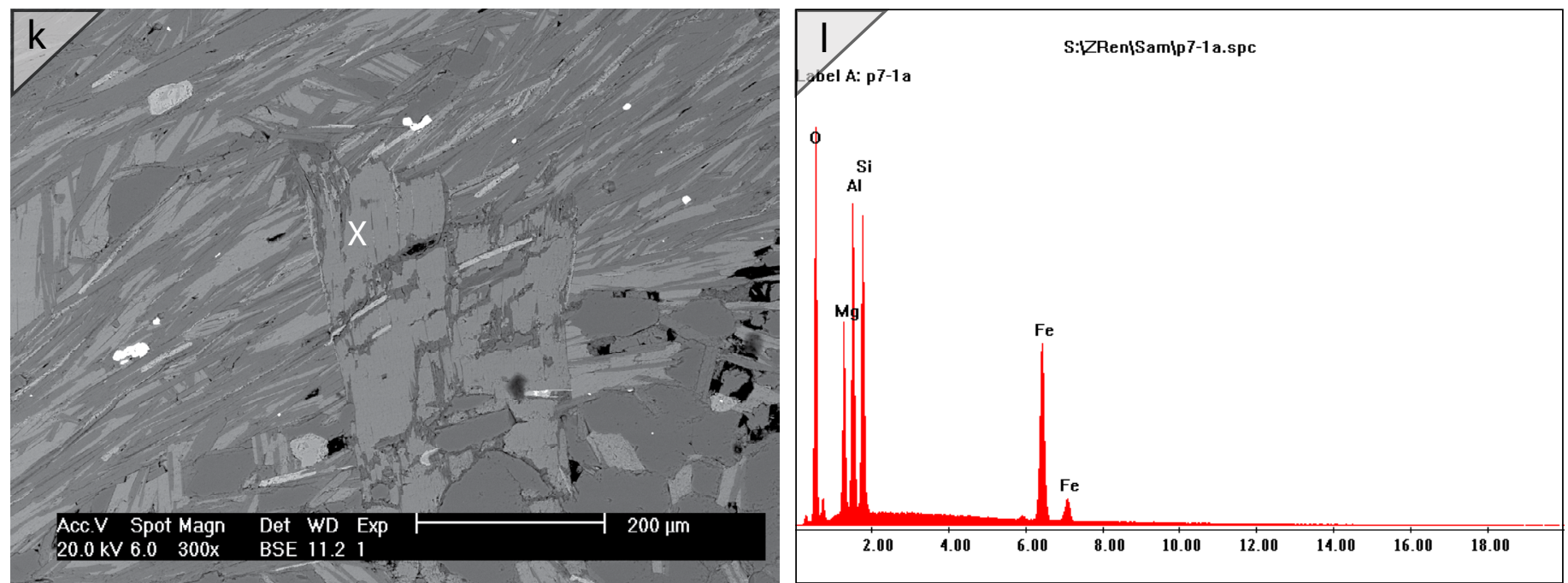

A2. k) SEM-BSE image of post tectonic chlorite.

A2. 1) SEM-EDS spectra of the chlorite in A2.k) 
Dunstan et al. 2016. Australin Journal of Earth Sciences 63

http://dx.doi.org/10.1080/08120099.2016.1131195
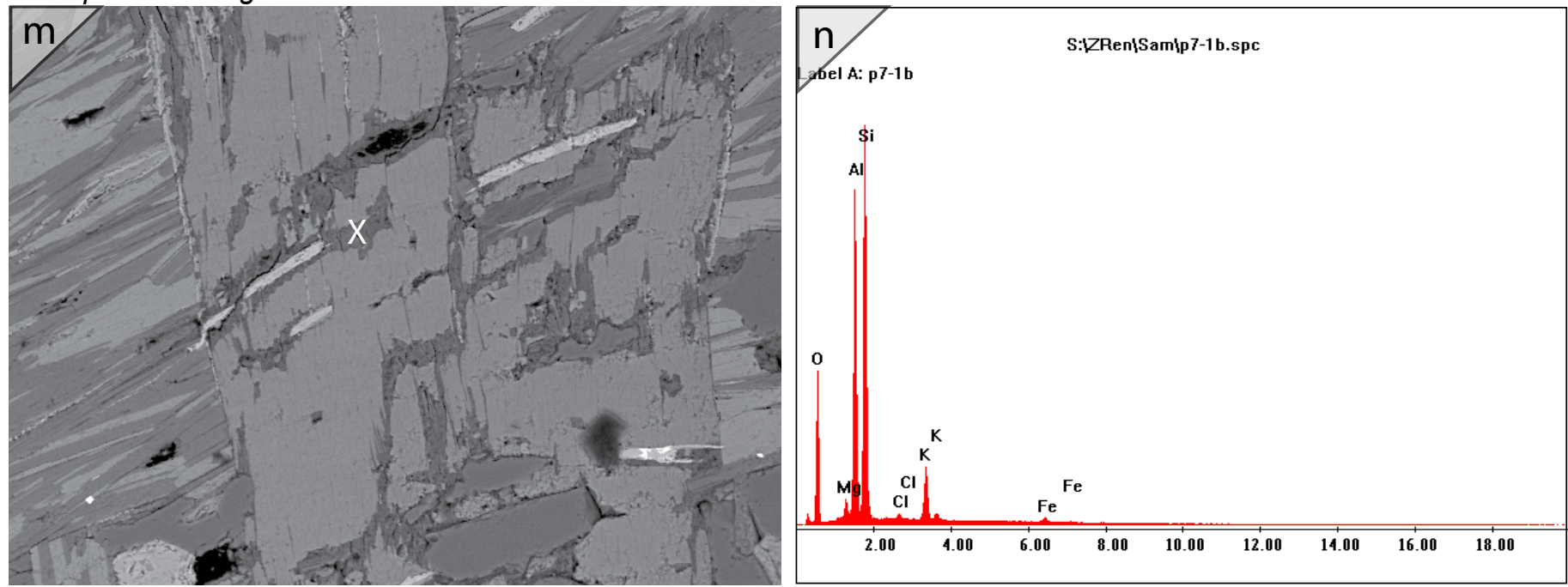

A2. m) SEM-BSE image of biotite within the post tectonic

A2. n) SEM-EDS spectra of the biotite in A2.m) chlorite in A2.k. 